\title{
79. A New Type of Skeletal Muscle Glycogenosis due to Phosphofructokinase Deficiency
}

\author{
By Mitsuo Nishikawa,*) Kazuo TsukiYama,*) \\ Takumi Enomoto, ${ }^{*)}$ Seiichiro TaruI, ${ }^{*)}$ Giichi OKuno, ${ }^{*}$ \\ Keishi UEdA, *) Tomoharu IkURA, *) Tsutomu TsuJII, *) \\ Toru Sugase, ${ }^{*}$ Masami SudA, ${ }^{* *}$ and Takehiko TANAKA**) \\ (Comm. by Arao Imamura, M.J.A., April 12, 1965)
}

Genetic defects of several enzymes which are directly or indirectly related to glycogen catabolism have been known: phosphorylase, amylo-1, 6-glucosidase, glucose-6-phosphatase, and acid maltase (suggested). Thus, glycogen storage diseases have been recently classified into six types, of which McArdle's disease has been given a special attention as one of the metabolic myopathies-muscle phosphorylase deficiency. It is, however, noteworthy that in spite of the variety of enzyme defects that are known, some cases of glycogen storage disease are still unexplained.

This report is concerned to three siblings suffering from a new type of skeletal muscle glycogenosis due to phosphofructokinase deficiency. The parents are first cousins and three siblings out of five are affected, a 20-year-old female (case 1) and 23- and 27-year-old male adults (case 2 and case 3 ). The most striking feature of the three patients is their inability to perform moderately severe muscular work. Since childhood, they have complained of easy fatiguability and have been unable to keep pace with other children. Forced exertion, such as climbing stairs or slope produced stiffness and pain in muscle groups of their legs, which lasted almost overnight and was accompanied by thirst, occasionally by headache and nausea.

On routine clinical examination, no abnormalities were found physically as well as mentally except slight disturbance of growth. Muscular tone and power were normal everywhere, with no sign of atrophy or hypertrophy. Histological examination of the muscle, too, showed no abnormal appearances on HE, PAS and carmine stainings.

Electromyographic studies revealed no obvious abnormalities except some high amplitude spikes observed in case 1 and 3 during voluntary contraction. Glucose tolerance curve was normal and injection of $1 \mathrm{mg}$ of glucagon produced a normal rise in blood sugar.

Ischemic forearm exercise test (Fig. 1): Blood lactate produced by exercise during ischemia was studied. Two sphygmomanometer

*) 2nd Department of Internal Medicine Osaka University School of Medicine.

**) Protein Institute Osaka University. 
Effect of ischaemic exercise

on the blood lactate level

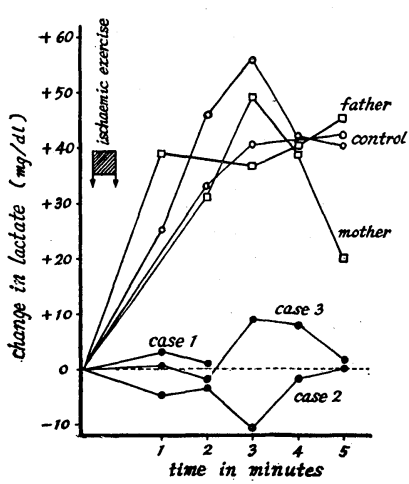

Fig. 1.

cuffs were inflated to $200 \mathrm{mmHg}$. which were applied around one wrist and the upperarm respectively. The subject then vigorously exercised the hand by squeezing a bulb of another manometer for fifty-five seconds. One minutes after terminating the exercise, the upperarm-cuff was deflated and successive venous blood specimens were taken from the cubital vein every one minutes for five minutes and lactate in each specimen was determined. The increase in blood lactate in both parents was approximately of the same degree as in the normal control, but no rise was observed in the three patients. And the patients developed severe contracture associated with pain in the muscles of the ischemic forearm and hand, which embarassed the patients during several hours. No electrical activities were recorded from the needle electrodes inserted into the shortened muscle, though electrical stimulation to the ulnar nerve could elicite action potential in the hypothenar muscle. From these findings, McArdle's disease was first suggested. Studies of muscle tissue in vitro, however, revealed normal activities of phosphorylase, indicating a block on some other point in the glycogenolytic pathway than in the case of McArdle's disease.

Biochemical studies of muscle tissue: Biochemical studies of the biopsied muscle tissues were systemically carried out. The amount of lactate formed on incubation of muscle homogenates without substrate, with addition of glycogen, glucose-1-phosphate, glucose-6-phosphate or fructose-6-phosphate was very small respectively, whereas addition of fructose-1, 6-diphosphate as substrate resulted in a increase of more than two to three times in the amount of lactate production as in the case of normal controls.

The glycogen content of the patients' muscles increased to 4.3, 1.5, and $2.6 \%$ and the structure was interpreted as normal from the degradation rate by beta-amylase. The content of glucose-6-phosphate and fructose-6-phosphate, also increased by more than five times, whereas the content of fructose-1, 6-diphosphate was very smallone twentieth or thirtieth of the control.

Determination of the enzyme activities in each step of glycolysis which were carried out thereafter (Table I), revealed that phosphofructokinase activity was almost absent, approximately one fiftieth of the control and the activities of phosphoglucomutase, phosphoglucoiromerase, aldolase as well as the other enzymes in glycolysis 
Table I. Levels of glycolytic enzymes in muscle specimens

\begin{tabular}{|c|c|c|c|c|c|c|}
\hline Enzymes & Case 1 & Case 2 & Case 31 & Father & Mother & Normals \\
\hline \multirow[t]{2}{*}{ Phosphorylase } & 25.8 & 18.6 & 21.6 & 21.4 & 15.6 & $19.0 \pm 2.8$ \\
\hline & \multicolumn{6}{|c|}{ inorg. $\mathrm{P}$ mg. liberated/10 min./g.w.w. } \\
\hline Phosphoglucomutase & 0.82 & 1.01 & 0.91 & 0.80 & 0.72 & $0.80 \pm 0.14$ \\
\hline Phosphoglucoisomerase & 0.81 & 0.95 & 0.54 & 0.49 & 0.36 & $0.57 \pm 0.19$ \\
\hline Phosphof ructokinase & 0.002 & 0.004 & 0.005 & 0.24 & 0.16 & $0.19 \pm 0.03$ \\
\hline Aldolase & 1.30 & 1.25 & 1.04 & 0.84 & 0.58 & $0.42 \sim 2.60$ \\
\hline $\begin{array}{l}\text { 3-Phosphoglyceroaldehyde- } \\
\text { dehydrogenase }\end{array}$ & & 1.25 & 0.35 & 0.29 & 0.23 & $0.19 \sim 0.80$ \\
\hline 3-Phosphoglycerate Kinase & & 0.87 & 0.68 & 0.87 & 0.44 & $0.50 \sim 0.62$ \\
\hline Phosphoglycerate Mutase & & 0.68 & 1.19 & 0.57 & 1.20 & $0.58 \sim 1.01$ \\
\hline Phosphoglycerate Enolase & & 0.46 & 0.23 & 0.21 & 0.10 & $0.09 \sim 0.31$ \\
\hline Pyruvate Kinase & 4.5 & 5.0 & 7.5 & 3.5 & 1.9 & $2.0 \pm 0.1$ \\
\hline \multirow[t]{2}{*}{ Lactic Dehydrogenase } & 1.30 & 0.40 & 1.52 & 0.81 & 0.64 & $0.98 \sim 1.76$ \\
\hline & \multicolumn{6}{|c|}{$\mu$ moles substrate converted $/ \mathrm{min} . / \mathrm{mg}$. protein } \\
\hline
\end{tabular}

were within the normal range. It was interesting that the activity of pyruvate kinase was rather higher than the control level, suggesting a compensation mechanism.

Thus, it has been clarified that this metabolic myopathy is a new clinical entity or a new type of skeletal muscle glycogenosis caused by phosphofructokinase deficiency. The patients suffer from a defect in glycogenolysis, limiting the energy available for muscular exertion to sources other than anerobic breakdown of glycogen.

Abnormalities in red blood corpuscules (R.B.C.): The activity of phosphofructokinase in tissues other than the muscle was investigated in R.B.C. The patients showed no anemia, but slight increase in reticulocyte counts and erythropoietic hyperplasia in the bone marrow were observed in all three. In case 2 , slight increase in serum bilirubin was also proved. The life span of R.B.C. in case 1 was shortened and the half life with ${ }^{51} \mathrm{Cr}$ labeled R.B.C. was reduced to approximately fourteen days. The osmotic fragility of R.B.C., however, was not decreased in all cases. The phosphofructokinase activities of the patients' R.B.C. were found to be partially deficient, which were of far less degree than in the muscles, approximately one third to one half of the control's R.B.C. It has been recognized that a slight acceleration of hemolysis caused by the disturbed glycolysis in R.B.C. exists in the patients.

Therapeutic effect of fructose: Several therapeutic measures for the muscular weakness have been tried and fructose appeared to be somewhat effective in every three cases. In case 1, she could maintain flexion-extension movements of the fingers with a frequency of once 
a second for about only forty times without any delay. After intravenous administration of fructose, however, she could repeat them 280 times without interruption. She could sustain a slow walking of 500 meters on the level ground before, and she could extend the distance to 750 meters after administration of fructose either per os or per injection. The mechanism of fructose effect has not been fully clarified.

\section{References}

McArdle, B.: Clin. Sci., 10, 13 (1951).

Schmid, R., and Mahler, R.: J. Clin. Invest., 38, 2044 (1959).

Thomson, W. H. S., MacLaurin, J. C., and Prineas, J. W.: J. Neurol. Neurosurg. Psychiat., 26, 60 (1963).

Tarui, S. et al.: In press (Biochem. Biophys. Res. Commun.). 